\title{
Effects of aspirin, vitamin D3, and progesterone on pregnancy outcomes in an autoimmune recurrent spontaneous abortion model
}

\author{
Yihong Chen ${ }^{1 * \oplus}$, Qiumei $\mathrm{Wu}^{2 * \oplus}$, Juanbing $\mathrm{Wei}^{1 \oplus \otimes}$, Jifen $\mathrm{Hu}^{1 \oplus \otimes}$, and Shan Zheng ${ }^{1 \oplus}$ \\ ${ }^{1}$ Department of Obstetrics and Gynecology, The First Affiliated Hospital of Fujian Medical University, Fuzhou, China \\ ${ }^{2}$ Department of Ultrasound, Fujian Provincial Maternal and Children's Hospital, Affiliated Hospital of Fujian Medical University, \\ Fuzhou, China
}

\begin{abstract}
High proportions of placental lymphocytes expressing DX5+/CD25+/FOXP3 +/CD45+/CD4 + are beneficial to maintain immune tolerance and improve pregnancy outcomes. This study aimed to compare and evaluate the therapeutic effects of aspirin, vitamin D3 (VitD3), and progesterone on the autoimmune recurrent spontaneous abortion (RSA) model. The autoimmune RSA mouse model was constructed, and the embryo loss rate was calculated for each group. Then, primary mouse placental lymphocytes were isolated, and the expression of DX5+/CD25+/FOXP3 +/CD45+/CD4 + was detected through flow cytometry. The serum levels of anti-cardiolipin antibody (ACA), $\beta 2-G P 1, C X C L 6$, IFN- $\gamma$, and IL- 6 were measured by ELISA to evaluate the proportion of Th1 and Th2 cells. Autoimmune RSA significantly increased the embryo loss rate, which was improved by aspirin, VitD3, and progesterone treatment, and progesterone treatment had the best effect among the three treatments. The positive expression of DX5 +/CD25 +/FOXP3 +/CD45+/CD4 + in the VitD3 and progesterone groups was significantly higher than that in the autoimmune RSA group, and the expression was highest in the progesterone treatment group. In the plasma of autoimmune RSA mice, the ACA, $\beta 2-G P 1$, CXCL6, and IFN- $\gamma$ levels were significantly higher and the IL-6 level was lower than the levels in control mice. All these changes could be reversed by aspirin and progesterone treatment. In conclusion, aspirin, VitD3 and progesterone treatment improved pregnancy outcomes in autoimmune RSA mice by regulating the Th1/Th2 balance and cytokines, and progesterone had the best effect of the three treatments.
\end{abstract}

Key words: Autoimmune RSA; Cytokines; Aspirin; Vitamin D3; Progesterone

\section{Introduction}

Spontaneous abortion is a common complication in the gestational period and is generally referred to as a pregnancy failure since it occurs before 28 weeks of gestation and fetal weight less than $1000 \mathrm{~g}(1,2)$. Recurrent spontaneous abortion (RSA) is the occurrence of spontaneous abortion at least three times in succession (3). Apart from chromosomal, anatomic, and endocrine abnormalities, approximately $50-60 \%$ of RSAs are related to immune factors in terms of its pathogenesis $(4,5)$. Patients with autoimmune spontaneous abortion have one or multiple autoantibodies, including anti-cardiolipin antibody (ACA), anti-zona pellucida antibody (AzpAb), anti-ovarian antibody (AoAb), and anti-endometrium antibody (EmAb) $(6,7)$. Among the autoantibodies, ACA is the most highly correlated with RSA, likely inducing placental thrombosis and obstruction, leading to abortion (8). Research indicates that
ACAs can bind with phospholipids on the vascular endothelial cell surface to form immune complex deposits and induce a series of inflammatory changes that ultimately lead to thrombosis $(9,10)$.

Aspirin, the primary antiplatelet drug, can irreversibly suppress epoxidase-1 activity in order to exert its antiplatelet aggregation effect. Aspirin can be hydrolyzed into salicylic acid and acetic acid in vivo and can effectively inhibit abortion of an embryo without causing any adverse reactions in pregnant women $(11,12)$. At present, the main treatment for autoimmune RSA is aspirin combined with heparin. Vitamin D3 (VitD3) is a type of steroid derivative that can be involved in immune regulation through cellular signal transduction pathways; in addition, VitD3 may also be related to T-lymphocyte differentiation and induction of immune tolerance, but its role in spontaneous abortion

Correspondence: Juanbing Wei: <weijbinggg@163.com > | Jifen Hu: <hujfen1199@163.com>

*These authors contributed equally to this work.

Received April 20, 2020 | Accepted November 23, 2020 
remains unclear $(13,14)$. Progesterone, a natural progestogen secreted by the ovary, is the first-line drug for clinical luteal support and confers obvious protection for the endometrium. Its common clinical indications include premature delivery, dysfunctional uterine bleeding, assisted reproduction after embryo transplantation, and luteal phase defects. Progesterone has no androgen activity, few side effects, and favorable clinical safety $(15,16)$.

In this study, anti- $\beta 2-G P 1$ antibodies and ACAs were induced by injecting mice with $\beta 2-G P 1$ to construct the autoimmune RSA mouse model (17). Based on the current understanding of that disease model, aspirin, VitD3, and progesterone were used to intervene in the pregnancy outcome of autoimmune RSA. Additionally, the potential effects, advantages, and disadvantages of the three drugs were also examined to provide a reference for further research.

\section{Material and Methods}

\section{Animals}

BALB/c mice (female, $20 \pm 2.05 \mathrm{~g}, 54-58$ days old; male, $26 \pm 3.42 \mathrm{~g}, 47-51$ days old) were purchased from Changsha SLAC Laboratory Animal Company [China, http://www.hnsja.com/; Certificate No. SCXK (Xiang) 2016-0002]. All animals were housed in cages with free access to food and water, and they were acclimated for 1 week of adaptive feeding under conditions of $24^{\circ} \mathrm{C}$ on a normal 12-h light/dark cycle (lights on at 7:00 AM) prior to experimental surgery to minimize animal suffering.

The animal protocol was approved by the Animal Care and Use Committee of The First Affiliated Hospital of Fujian Medical University and was consistent with the National Institutes of Health Guide for the Care and Use of Laboratory Animals.

\section{Reagents}

32-GP1 was purchased from Novoprotein (Cat. No. DC432, USA). Incomplete Freund's adjuvant (IFA) (Cat. No. 642862) and complete Freund's adjuvant (CFA) (Cat. No. 642852) were purchased from Solarbio (China). Progesterone was purchased from Solarbio (Cat. No. SP9440). Aspirin enteric-coated tablets were purchased from Bayer HealthCare (Cat. No. J20171021, USA). Vitamin D3 was purchased from Solarbio (Cat. No. V8070). A mouse spleen lymphocyte separation reagent kit was purchased from TBD Sciences (LTS1092PK, China). A mouse $\beta 2-$ GPI ELISA kit (Cat. No. MM-0951 M1), mouse interferon (IFN)- $\gamma$ ELISA kit (Cat. No. MM-0182 M1), mouse ACA ELISA kit (Cat. No. MM-0583 M1), mouse interleukin (IL)-6 ELISA kit (Cat. No. MM-0163 M1), and mouse CXCL6 ELISA kit (Cat. No. MM-0950 M1) were all purchased from Meimian Biotechnology (China). CD4-FITC (Cat. No. 100405), CD25-PE/CY7 (Cat. No. 102015), CD49b-PE (Cat. No. 108907), FOXP3-Alexa Fluor 647 (Cat. No. 320013), CD45-Percp/CY5.5 (Cat. No.
103131), fixation buffer (Cat. No. 420801), and intracellular staining permeabilization wash buffer (Cat. No. 421002) were all purchased from BioLegend (USA).

\section{Autoimmune RSA mouse model}

The autoimmune RSA mouse model was constructed according to the method described by Xiao et al. (7). Female mice were given intrauterine injections of $50 \mu \mathrm{L}$ PBS containing $10 \mu \mathrm{g}$ human $\beta 2-\mathrm{GP} 1$ and $10 \mu \mathrm{g}$ CFA for 1 week. Then, CFA was replaced with IFA, and the same dose was administered. After 10 days of injections, female mice were housed with male mice at a ratio of 2:1. From the date that cohabitation began, mice were observed at two fixed time periods in the morning and in the afternoon. If a vaginal plug was observed, the female was raised in an individual cage and was used for the autoimmune spontaneous abortion mouse model; the non-pregnant female mice were excluded. The day of vaginal plug detection was considered day 0 of pregnancy. A total of 35 pregnant female mice were randomly divided into 5 groups ( $n=7$ for each group): the normal pregnant group (Control), the model group (Model, autoimmune RSA mice given distilled water by oral gavage at $0.1 \mathrm{~mL} /$ $10 \mathrm{~g}$ once a day for 14 days continuously after the vaginal plug was observed), the aspirin group (Aspirin, autoimmune RSA mice given aspirin by oral gavage at $0.0195 \mathrm{mg} / \mathrm{g}$ once a day for 14 days continuously from the first day of gestation), the vitamin D3 group (VitD3, autoimmune RSA mice given VitD3 by intraperitoneal injection at $4 \mu \mathrm{g} / \mathrm{mouse}$ once a day for 14 days continuously from the first day of gestation), and the progesterone group (Progesterone, autoimmune RSA mice given progesterone by intraperitoneal injection at $4 \mathrm{mg} / \mathrm{kg}$ once a day for 14 days continuously from the first day of gestation).

On day 15 of gestation, blood samples $(1 \mathrm{~mL})$ were collected from the mouse abdominal aorta under anesthesia. Then, mice were sacrificed by cervical dislocation, and uterine horns were isolated from pregnant mice. The placental weight, fetal weight, and total number of embryos lost were recorded. The embryo loss rate was calculated according to the following formula: embryo loss rate $(\%)=$ (number of lost fetuses / number of fetuses) $\times 100$.

\section{Placental lymphocyte isolation}

The pregnant mice were sacrificed, disinfected with ethanol for $5 \mathrm{~min}$, and dissected on the benchtop to collect the placentas. Afterwards, the placentas were cut into pieces and washed with Hanks' buffer 2-3 times. Then, the upper layer of the cell suspension was discarded, and $0.1 \%$ collagenase I was added into stop buffer to digest the tissue for $30 \mathrm{~min}$, followed by agitation. The above steps were repeated several times until the placental tissues were completely digested. The cell suspension was filtered using a $70 \mu \mathrm{m}$ sieve, $0.125 \%$ trypsin was used to digest the cells for 1-2 $\mathrm{min}$, the suspension 
underwent centrifugation at $1480 \mathrm{~g}$ for $5 \mathrm{~min}$ at $4^{\circ} \mathrm{C}$, and the monocytes were isolated using mouse visceral lymphocyte separating medium.

\section{Detection of DX5 + /CD25 + /FOXP3 + /CD45 +/CD4 + expression in placental lymphocytes by flow cytometry}

A total of $100 \mu \mathrm{L}$ cell suspension was collected in an Eppendorf tube, then cells were incubated with $5 \mu \mathrm{L}$ each CD4-FITC, CD25-PE/CY75, CD49b-PE, and CD45Percp/CY5.5 in the Eppendorf tube for 10 min in the dark. Afterwards, cells were washed in $1 \mathrm{~mL}$ PBS and fixed in $500 \mu \mathrm{L}$ fixing agent for $30 \mathrm{~min}$. Cells were then collected and washed in $1 \mathrm{~mL}$ PBS and incubated in $1 \mathrm{~mL}$ permeabilization reagent for $30 \mathrm{~min}$ in the dark. Subsequently, the cells were washed again and resuspended in $100 \mu \mathrm{L}$ PBS with $5 \mu \mathrm{L}$ FOXP3-Alexa Fluor 647 and incubated for $10 \mathrm{~min}$ in the dark. Cells were washed again and incubated in $500 \mu \mathrm{L}$ PBS for analysis by flow cytometry (NovoCyte 2060R, Acea Biosciences, China). A total of 10,000 cells were screened and analyzed automatically by the flow cytometer, and the operator was blind to the group assignment of the cells.

\section{Enzyme-linked immunosorbent assay (ELISA)}

The plasma levels of ACA, $\beta 2-G P 1$, CXCL6, IFN- $\gamma$, and IL-6 were determined using ELISA. In brief, blood samples $(1 \mathrm{~mL})$ were collected through the mouse's heart under deep anesthesia and then underwent 15 min of centrifugation at $800 \mathrm{~g}$ at $4^{\circ} \mathrm{C}$. Then, the plasma IL-1 $\beta$ concentration was determined using commercially available ELISA kits in accordance with the manufacturer's instructions. Absorbance was measured at $450 \mathrm{~nm}$ using a microplate reader (RT-6100, Rayto Life and Analytical Sciences, China). The concentrations of each cytokine were calculated according to the standards and reported as picograms per milligram (pg/mg).

\section{Statistical analysis}

Three repeated experiments were conducted for each set of measurements, and the resulting data are reported as means $\pm S D$. The statistical package SPSS 18.0 (IBM, USA) was used for all statistical analyses. The data were analyzed using one-way analysis of variance (ANOVA) followed by Dunnett's test. $\mathrm{P}<0.05$ was considered statistically significant.

\section{Results}

\section{Detection of embryo loss rate}

Pregnancy outcomes are shown in Figure 1. There were no significant differences in the placental weight and fetal mouse weight among the groups. Autoimmune RSA significantly increased the embryo loss rate, which was improved by treatment with aspirin, VitD3, and progesterone; progesterone treatment had the best effect.

\section{Detection of DX5 + /CD25 + /FOXP3 + /CD45 +/CD4 + expression in placental lymphocytes}

The high percentage of DX $5+/ C D 25+/ F O X P 3+/ C D$ $45+/ C D 4+-$ positive placental lymphocytes is beneficial to maintain immune tolerance and improve pregnancy outcomes (18-20). As shown in Figure 2, the proportion of DX5 + /CD25 + /FOXP3 +/CD45 + /CD4 + -positive cells in the model group was significantly lower than that in the control group $(P<0.05)$; the proportion of positive cells was significantly higher in the VitD3 and progesterone groups than in the model group $(\mathrm{P}<0.05)$. Relative to the model group, the DX5 +/CD25 +/FOXP3 +/CD45 +/CD4 + proportion in the aspirin group was not significantly increased. The proportion of positive cells in the progesterone group was the most significantly increased from the model group, but no obvious difference was detected between the progesterone group and the control group.
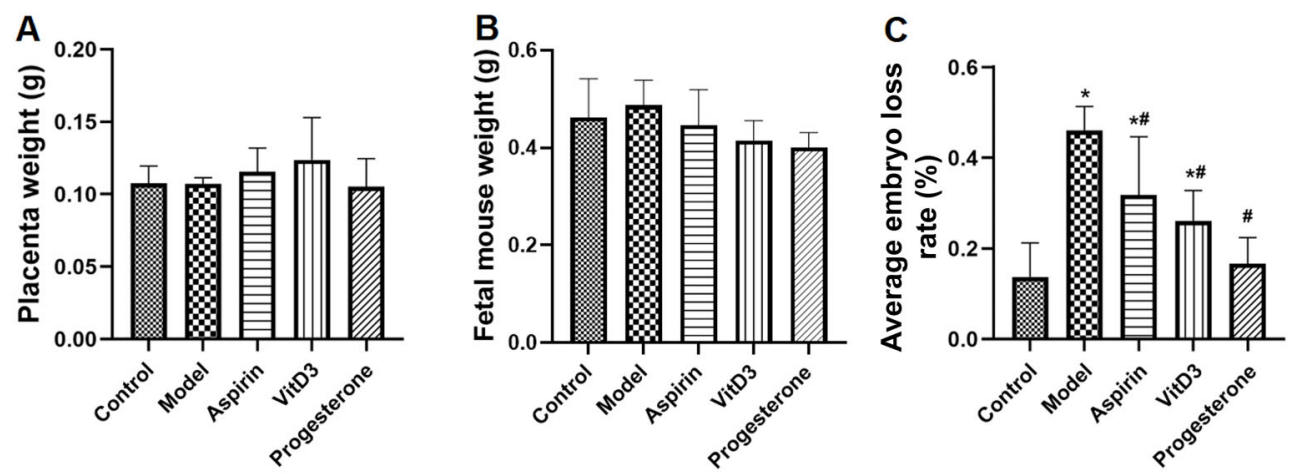

Figure 1. Effects of aspirin, vitamin D3 (VitD3), and progesterone on the placental weight (A), fetal mouse weight (B), and embryo loss rate $(\mathbf{C})$ in autoimmune recurrent spontaneous abortion mice. Data are reported as means $\pm S D(n=5)$. ${ }^{*}<0.05$ vs the control group; ${ }^{\#} \mathrm{P}<0.05$ vs the model group (ANOVA). 

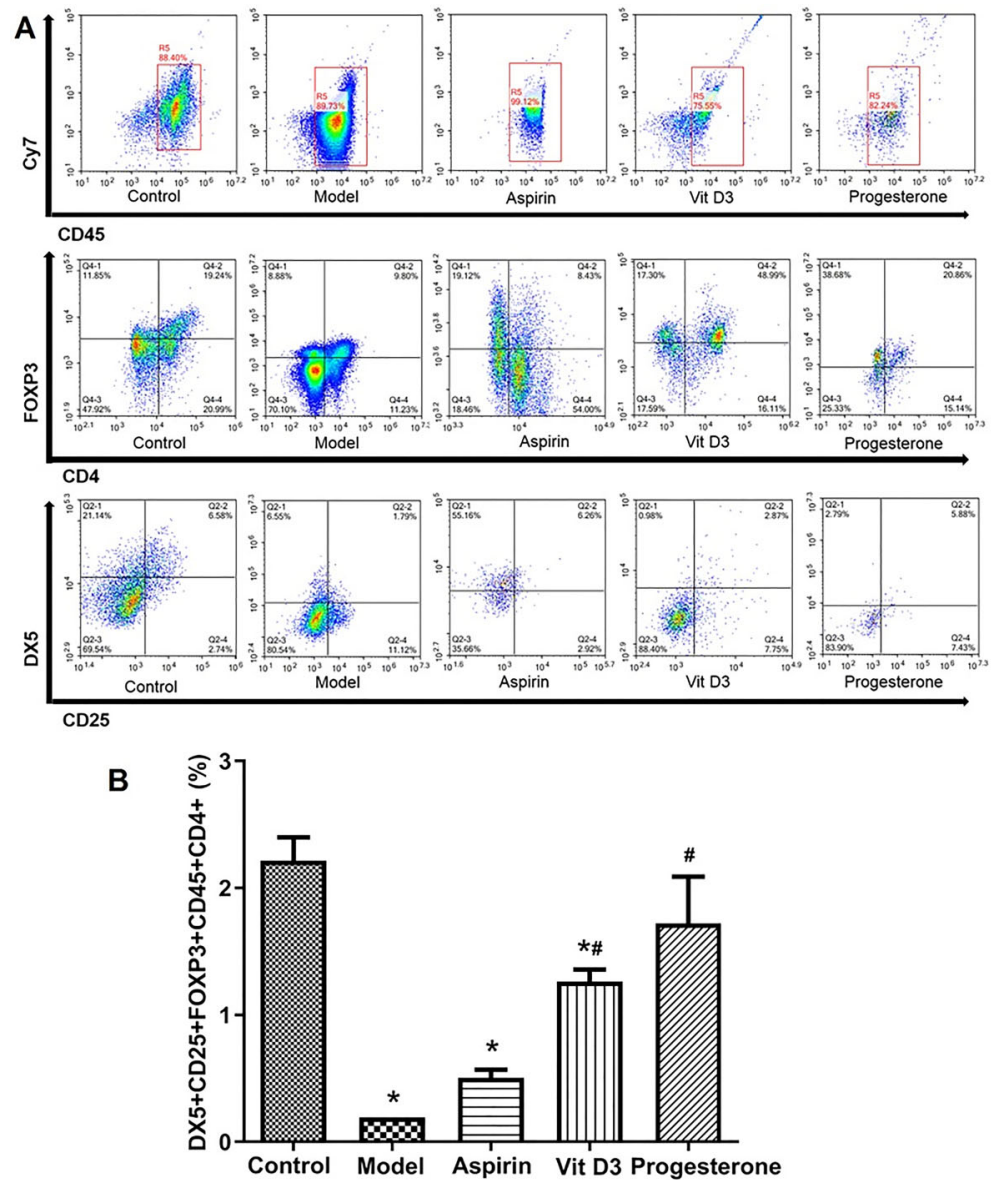

Figure 2. The expression of DX5 $+/ C D 25+/ F O X P 3+/ C D 45+/ C D 4+$ in placental lymphocytes in a mouse model of recurrent spontaneous abortion treated with aspirin, vitamin D3, and progesterone was detected by flow cytometry. A, Placental lymphocytes were collected and stained with relevant markers for flow cytometry analysis. B, Quantitative analysis of the percentage of DX5 $+/ C D 25+/$ FOXP3 +/CD45+/CD4 + cells in each group. Data are reported as means $\pm S D(n=3)$. ${ }^{*} P<0.05$ vs the control group; ${ }^{\#} P<0.05$ vs the model group (ANOVA).

\section{Detection of Th cells in peripheral blood}

The Th1/Th2 balance is directly related to pregnancy outcomes; therefore, Th1 and Th2 cells in the peripheral blood were analyzed by flow cytometry. As shown in Figure 3 , among CD3 + /CD4 + positive cells, IFN- $\gamma$-positive cells were Th1 cells, and IL-4-positive cells were Th2 cells. The ratio of Th1/Th2 was significantly decreased in the autoimmune RSA model group, indicating an imbalance of Th1/ Th2, which was improved by treatment with aspirin, VitD3, and progesterone. The progesterone treatment had the best effect, and the Th1/Th2 ratio was even better than that in the normal control group.

\section{Detection of serum ACA and $\beta 2-$ GP1 antibody levels}

High expression levels of ACA and $\beta 2-G P 1$ have been reported to be closely associated with RSA (21). As shown in Figure 4, the ACA and $32-G P 1$ levels in the model group were significantly higher than those in the control group
$(\mathrm{P}<0.05)$; the antibody levels in the three treatment groups were lower than those in the model group, and the levels in the progesterone group were significantly lower than those in the model group $(\mathrm{P}<0.05)$.

\section{Detection of serum CXCL6, IFN- $\gamma$, and IL-6 cytokines levels}

To evaluate the Th1/Th2 balance, Th1 cytokines (CXCL6 and IFN- $\gamma$ ) and a Th2 cytokine (IL-6) were detected in serum using ELISA $(22,23)$. As shown in Figure 5, the CXCL6 and IFN- $\gamma$ levels in the model group were significantly lower than those in the control group $(\mathrm{P}<0.05)$. Compared with the model group, the CXCL6 and IFN- $\gamma$ levels in the three medication groups had the most significant decreases in the cytokines measured $(\mathrm{P}<0.05)$. The IL-6 protein level in the model group was significantly lower than that in the control group $(P<0.05)$, while the IL-6 levels in the medication groups 

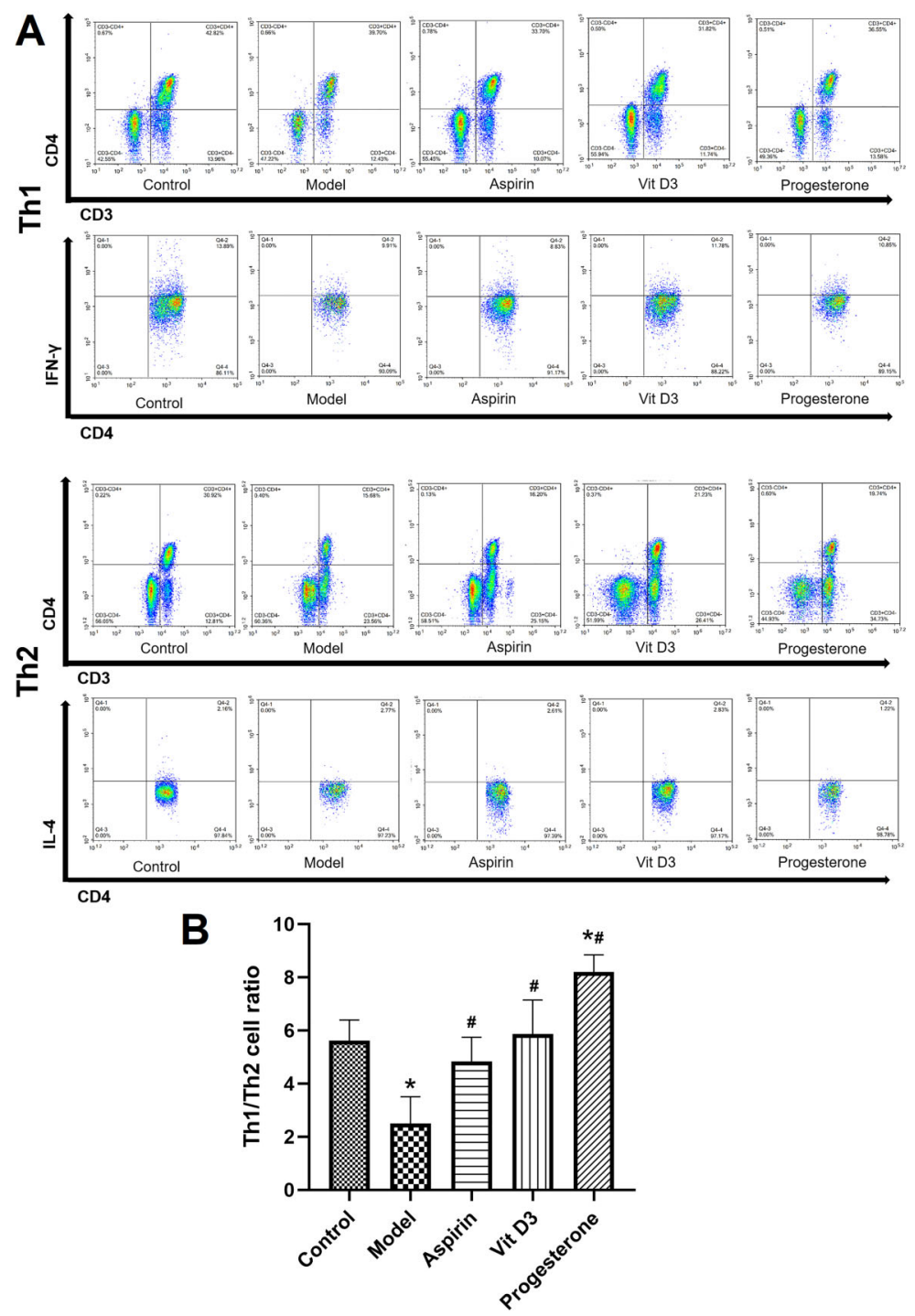

Figure 3. Th1 and Th2 cells in the peripheral blood in a mouse model of recurrent spontaneous abortion treated with aspirin, vitamin D3, and progesterone were analyzed by flow cytometry. A, Representative flow cytometry results. B, Quantitative analysis of the percentage of Th1 and Th2 cells in each group. Data are reported as means $\pm S D(n=3) .{ }^{*} \mathrm{P}<0.05$ vs the control group; ${ }^{\#} \mathrm{P}<0.05$ vs the model group (ANOVA).
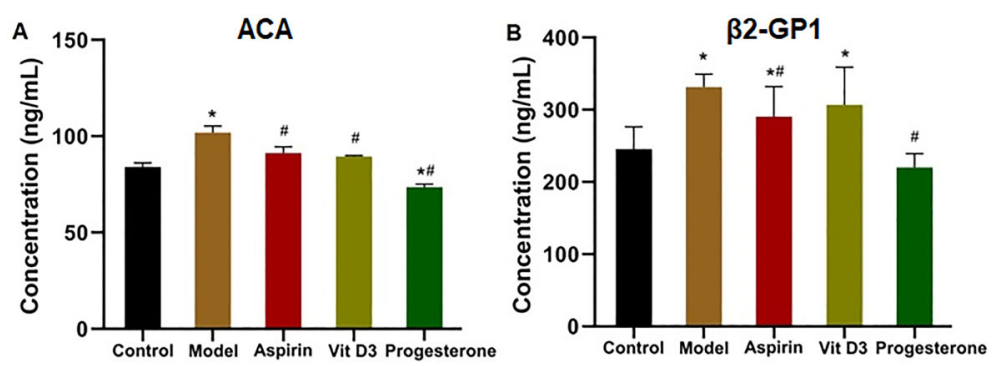

Figure 4. The anti-cardiolipin antibody (ACA) and $\beta 2-\mathrm{GP} 1$ levels in a mouse model of recurrent spontaneous abortion treated with aspirin, vitamin D3, and progesterone were detected by ELISA. Data are reported as means $\pm S D(n=3)$. ${ }^{*}<0.05$ vs the control group; ${ }^{\#} \mathrm{P}<0.05$ vs the model group (ANOVA). 

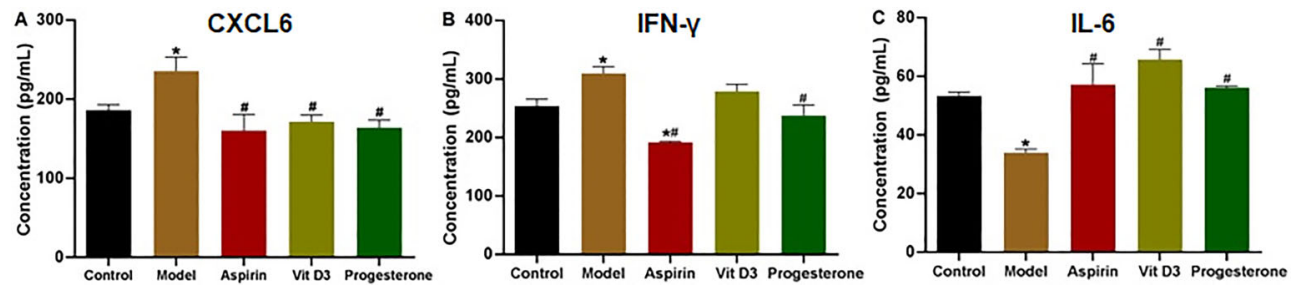

Figure 5. The cytokines in the serum of a mouse model of recurrent spontaneous abortion treated with aspirin, vitamin D3, and progesterone were detected using ELISA. The CXCL6, interferon (IFN)- $\gamma$, and interleukin (IL)- 6 levels in the serum in each group were analyzed by ELISA. Data are reported as means $\pm S D(n=3)$. ${ }^{*} P<0.05$ vs the control group. ${ }^{\#} P<0.05$ vs the model group.

were significantly higher than those in the model group $(P<0.05)$.

\section{Discussion}

The pathogenesis of autoimmune RSA remains incompletely understood at present, although it is generally believed to be related to the expression of multiple antibodies $(24,25)$. Currently, there are multiple exploratory treatments, but the results of these treatments are partially contradictory. In this study, aspirin, VitD3, and progesterone were used to intervene in the autoimmune RSA pregnancy outcome, and the effects of these three drugs were compared between groups.

First, the DX5 + /CD25 + /FOXP3 + /CD45 +/CD4 + expression in placental lymphocytes was detected through flow cytometry. CD45 is a leukocyte common antigen and is generally used as a leukocyte marker (26). CD4+ $\mathrm{FOXP3}+\mathrm{T}$ regulatory (Treg) cells are key players in suppressing autoimmunity and maintaining self-immune tolerance (27). DX5 + and CD25 + represent NK cell subsets that contribute to the establishment of immune tolerance (18). A high proportion of these cells is beneficial for maintaining immune tolerance and improving pregnancy outcomes (18-20). In our current study, we found that the VitD3 and progesterone groups had significantly higher proportions of DX5 +/CD25 +/FOXP3 + / CD $45+/ C D 4+$ cells than the model group, and progesterone had a better effect than VitD3.

At present, the Th1/Th2 balance is generally believed to be crucial in establishing immune tolerance at the maternal-embryonic interface. Researchers have found a shift in the Th1/Th2 equilibrium towards Th1 dominance in patients with recurrent miscarriage (28). Th1 cells mainly produce proinflammatory cytokines such as tumor necrosis factor- $\alpha$, IFN- $\gamma$, and IL-2, and these cytokines can stimulate cellular inflammation and participate in cellular immunity and the rejection process (29). Conversely, the Th2 cytokines, mainly IL-4, IL-6, IL-10, etc., activate the production of B-cell antibodies and function antagonistically to Th1 cytokines. Th2 cells can act as mediators of humoral immunity and participate in immune tolerance at the maternal-embryonic interface $(30,31)$. Th1 cytokines are dominant in autoimmune RSA (32). The positive expression of DX5+/CD25+/FOXP3 +/CD45+/CD4 + in cells was significantly elevated after treatment with progesterone or VitD3, indicating an increase in Th2 cells and that the Th1/Th2 imbalance was improved compared with that of the model group. The increased Th2 cells could enhance immune tolerance, which could ameliorate autoimmune RSA.

Furthermore, the ACA, $\beta 2-\mathrm{GP} 1, \mathrm{CXCL} 6, \mathrm{IFN}-\gamma$, and IL-6 levels in serum were detected by ELISA. ACAs are a group of negatively charged antiphospholipid antibodies; according to prior studies, the ACA positive rate in RSA patients was remarkably higher than that in healthy pregnant women, and ACA can bind with platelet phospholipids, which decreases release of prostacyclin and results in thrombosis (33). Based on our experimental results, three drugs could partially reduce ACA levels and suppress thrombosis. $32-\mathrm{GP} 1$, a single strand glycoprotein, is abundant in plasma and can bind with plasma lipoproteins to expose a concealed epitope, which leads to the production of anti- $\beta 2-G P 1$ antibody. The anti- $\beta 2-G P 1$ antibody has been experimentally verified to suppress the endogenous coagulation pathway and inhibit prothrombinase activity on platelets, giving rise to RSA (34). According to our results, aspirin and progesterone notably suppressed the anti- $\beta 2-G P 1$ antibody. The levels of Th1 factors CXCL6 and IFN- $\gamma$ in the medication groups were lower than those in the model group, while those of Th2 factor IL-6 were elevated, suggesting that Th2 cells were increased and immune tolerance was enhanced $(35,36)$. Consequently, aspirin, VitD3, and progesterone had various impacts and therapeutic effects on the autoimmune RSA model, and progesterone had the best effect.

At present, the main treatment for autoimmune RSA is heparin combined with aspirin. In addition to anticoagulation, heparin can also suppress the binding of $\beta 2-G P 1$ with the anti-phospholipid antibody, and aspirin can limit the production of thromboxane A2 and relieve vasoconstriction $(37,38)$. In our current study, we found that progesterone alone had the best effect on reducing the embryo loss rate and improving the Th1/Th2 balance. Furthermore, in the autoimmune RSA mice, the plasma ACA, $32-G P 1$, CXCL6, and IFN- $\gamma$ levels were significantly higher and the 
IL-6 level was lower than in the control mice. All these changes were reversed by aspirin and progesterone treatment. Previous study has found that a combination treatment consisting of oral prednisone $20 \mathrm{mg} /$ day and progesterone $20 \mathrm{mg} /$ day for the first 12 weeks of gestation, and aspirin $75 \mathrm{mg} /$ day for 32 weeks of gestation could improve the pregnancy outcomes (39). However, there was not enough evidence to show the effect of aspirin/progesterone or aspirin/vitamin D combination on the pregnancy outcomes. In addition, compared with aspirin, progesterone has widely been used clinically for

\section{References}

1. Kuon RJ, Wallwiener LM, Germeyer A, Strowitzki T, Daniel $\mathrm{V}$, Toth B. Establishment of a standardized immunological diagnostic procedure in RM patients. J Reprod Immunol 2012; 1: 55, doi: 10.1016/j.jri.2012.03.338.

2. Xiao S, Zhao A. The etiology of recurrent spontaneous abortion research. Chin J Gynecol Obstet 2014; 30: 41-45.

3. van den Berg MM, van Maarle MC, van Wely Mand, Goddijn M. Genetics of early miscarriage. Biochim Biophys Acta 2012; 1822: 1951-1959, doi: 10.1016/j.bbadis.2012. 07.001.

4. Elmahashi MO, Elbareg AM, Essadi FM, Ashur BM and Adam I. Low dose aspirin and low-molecular-weight heparin in the treatment of pregnant Libyan women with recurrent miscarriage. BMC Res Notes 2014; 7: 23, doi: 10.1186/ 1756-0500-7-23.

5. Franssen MT, Musters AM, van der Veen F, Repping S, Leschot NJ, Bossuyt PM, et al. Reproductive outcome after PGD in couples with recurrent miscarriage carrying a structural chromosome abnormality: a systematic review. Hum Reprod Update 2011; 17: 467-475, doi: 10.1093/ humupd/dmr011.

6. Feng YI, Lian G, XQ Li XM. Clinical analysis of 16 cases with duplex uterus. J Reprod Contracep 2011; 22: 217-221, doi: 10.1016/S1001-7844(12)60018-2.

7. Xiao SJ, Zhao AM, Bao SM. Relationship between expression of COX-2, its signal transduction pathways and autoimmune-type recurrent miscarriage. J Shanghai Jiaotong Univers (Medical Science) 2011; 7: 895-899.

8. Reed JR, Leon RP, Hall MK, Schwertfeger KL. Interleukin1 beta and fibroblast growth factor receptor 1 cooperate to induce cyclooxygenase-2 during early mammary tumourigenesis. Breast Cancer Res 2009; 11: R21, doi: 10.1186/ bcr2246.

9. Toth B, Jeschke U, Rogenhofer N, Scholz C, Würfel W, Thaler $\mathrm{CJ}$, et al. Recurrent miscarriage: current concepts in diagnosis and treatment. J Reprod Immunol 2010; 85: 2532, doi: 10.1016/j.jri.2009.12.006.

10. Zhang BY, Wei YS, Niu JM, Li Y, Miao ZL, Wang ZN. Risk factors for unexplained recurrent spontaneous abortion in a population from southern China. Int J Gynecol Obstet 2010; 108: 135-138, doi: 10.1016/j.ijgo.2009.09.019.

11. Drakeley AJ, Roberts D, Alfirevic Z. Cervical cerclage for prevention of preterm delivery: meta-analysis of randomized trials. Obstet Gynecol 2003; 102: 621-627, doi: 10.1016/ S0029-7844(03)00673-2. the treatment of gynecological diseases with little toxicity and few side effects. Further studies will focus on the safety and efficacy of the combination of aspirin and progesterone or VitD3 on autoimmune RSA.

\section{Acknowledgments}

This study was supported by the Training Project of Young Talents in Health System of Fujian Province (2017-ZQN-47) and the Qihang Fund of Fujian Medical University (2018QH1058).

12. Triolo G, Ferrante A, Ciccia F, Accardo-Palumbo A, Perino A, Castelli $A$, et al. Randomized study of subcutaneous low molecular weight heparin plus aspirin versus intravenous immunoglobulin in the treatment of recurrent fetal loss associated with antiphospholipid antibodies. Arthritis Rheum 2003; 48: 728-731, doi: 10.1002/art.10957.

13. Lei GS, Zhang C, Zimmerman MK, Lee CH. Vitamin D as supplemental therapy for Pneumocystis pneumonia. Antimicrob Agents Chemother 2015; 60: 1289-1297, doi: 10.1128/AAC.02607-15.

14. Shih EM, Mittelman S, Pitukcheewanont P, Azen CG, Monzavi R. Effects of vitamin D repletion on glycemic control and inflammatory cytokines in adolescents with type 1 diabetes. Pediatr Diabetes 2016; 17: 36-43, doi: 10.1111/ pedi.12238.

15. Wu G, Chen J, Hu X, Zhou H, Liu J, Lv D, et al. Pharmacokinetic properties of three forms of vaginal progesterone administered in either single or multiple dose regimen in healthy post-menopausal Chinese women. Front Pharmacol 2017; 8: 212, doi: 10.3389/fphar.2017.00212.

16. Posaci C, Smitz J, Camus M, Osmanagaoglu K, Devroey P. Progesterone for the luteal support of assisted reproductive technologies: clinical options. Hum Reprod 2000; 15: 129-148, doi: 10.1093/humrep/15.suppl 1.129.

17. He M, Zhou Y, Jiang M, Li F, Yang M, Fan Y, et al. Increased toll-like receptor-myeloid differentiation factor 88 expression at the maternal-fetal interface is associated with spontaneous abortion. Gynecol Obstet Invest 2017; 82: 553-562, doi: $10.1159 / 000468931$.

18. Ebata K, Shimizu Y, Nakayama Y, Minemura M, Murakami J, Kato T, et al. Immature NK cells suppress dendritic cell functions during the development of leukemia in a mouse model. J Immunol 2006; 176: 4113-4124, doi: 10.4049/ jimmunol.176.7.4113.

19. Scholzen A, Mittag D, Rogerson SJ, Cooke BM, Plebanski M. Plasmodium falciparum-mediated induction of human CD25hiFoxp3hi CD4 T cells is independent of direct TCR stimulation and requires IL-2, IL-10 and TGF $\beta$. PLoS Pathog 2009; 5: e10000543, doi: 10.1371/journal.ppat.1000543.

20. Lin $Y$, Chen $Y$, Zeng $Y$, Wang T, Zeng S. Lymphocyte phenotyping and NK cell activity analysis in pregnant NOD/ SCID mice. J Reprod Immunol 2005; 68: 39-51, doi: 10. 1016/j.ji.2005.05.002.

21. Lin QD, Qiu LH. Pathogenesis, diagnosis, and treatment of recurrent spontaneous abortion with immune type. Front 
Med China 2010; 4: 275-279, doi: 10.1007/s11684-0100101-y.

22. Chen Z, O'Shea JJ. Th17 cells: a new fate for differentiating helper T cells. Immunol Res 2008; 41: 87-102, doi: 10.1007/ s12026-007-8014-9.

23. Kwak-Kim J, Lee SK, Gilman-Sachs A. Elevated Th1/Th2 cell ratios in a pregnant woman with a history of RSA, secondary Sjögren's syndrome and rheumatoid arthritis complicated with one fetal demise of twin pregnancy. $A m \mathrm{~J}$ Reprod Immunol 2007; 58: 325-329, doi: 10.1111/j.16000897.2007.00506.x.

24. Wang S, Cao C, Piao H, Li Y, Tao Y, Zhang X, et al. Tim-3 protects decidual stromal cells from toll-like receptormediated apoptosis and inflammatory reactions and promotes Th2 bias at the maternal-fetal interface. Sci Rep 2015; 5: 9013, doi: 10.1038/srep09013.

25. Tian T, Sun D, Wang P, Wang H, Bai X, Yang X, et al. Roles of toll-like receptor 7 and 8 in prevention of intrauterine transmission of hepatitis B virus. Cell Physiol Biochem 2015; 37: 445-453, doi: 10.1159/000430367.

26. Rheinländer A, Schraven B, Bommhardt U. CD45 in human physiology and clinical medicine. Immunol Lett 2018; 196: 22-32, doi: 10.1016/j.imlet.2018.01.009.

27. Josefowicz SZ, Lu LF, Rudensky AY. Regulatory T cells: mechanisms of differentiation and function. Annu Rev Immunol 2012; 30: 531-564, doi: 10.1146/annurev.immu nol.25.022106.141623.

28. Beaman KD, Ntrivalas E, Mallers TM, Jaiswal MK, KwakKim J, Gilman-Sachs A. Immune etiology of recurrent pregnancy loss and its diagnosis. Am J Reprod Immunol 2012; 67: 319-325, doi: 10.1111/j.1600-0897.2012.01 118.x.

29. Liu J, Dong P, Wang S, Li J. Natural killer, natural killer T, helper and cytotoxic $T$ cells in the decidua from recurrent spontaneous abortion with normal and abnormal chromosome karyotypes. Biochem Biophys Res Commun 2019; 508: 354-360, doi: 10.1016/j.bbrc.2018.11.156.

30. Cocksedge K, Saravelos S, Wang Q, Tuckerman E, Laird S, $\mathrm{Li}$ T. Does free androgen index predict subsequent pregnancy outcome in women with recurrent miscarriage? Hum Reprod 2008; 23: 797-802, doi: 10.1093/humrep/den022.
31. Kheshtchin N, Gharagozloo M, Andalib A, Ghahiri A, Maracy MR, Rezaei A. The expression of Th1-and Th2-related chemokine receptors in women with recurrent miscarriage: the impact of lymphocyte immunotherapy. Am J Reprod Immunol 2010; 64: 104-112, doi: 10.1111/j.1600-0897.20 10.00829.x.

32. Ali S, Majid S, Niamat Ali M, Taing S. Evaluation of T cell cytokines and their role in recurrent miscarriage. Int Immunopharmacol 2020; 82: 106347, doi: 10.1016/j.intimp. 2020.106347.

33. Cao WL, Ye LL, Jiang W. Assessment on immunity factors and anti-immunity treat effect of recurrent spontaneous abortion [in Chinese]. Mater Child Health Care China 2010; 51.

34. Wang YF, Qin WB, Yang N, Cui R, Wang XL, Wang YX. Relationship between anti-HLA-I/II antibodies, anticardiolipin antibodies, anti- $\beta 2$ GP1 antibodies and infertility, recurrent spontaneous abortion [in Chinese]. China Prac Med 2013; 8: 35-36, doi: 10.3969/j.issn.1673-7555.2013.17.018 http://en. cnki.com.cn/Article_en/CJFDTOTAL-ZSSA201317019.htm.

35. Gore M, Sadosky A, Stacey BR, Tai KS, Leslie D. The burden of chronic low back pain: clinical comorbidities, treatment patterns, and health care costs in usual care settings. Spine 2012; 37: E668-E677, doi: 10.1097/BRS. Ob013e318241e5de.

36. Check JH. A practical approach to the prevention of miscarriage: part 2--active immunotherapy. Clin Exp Obstet Gynecol 2010; 37: 5-9.

37. Zhang G, Cheng LH. Low molecular weight heparin combined with low-dose aspirin could be effective in treatment of recurrent spontaneous abortion. Genomics Appl Biol 2017; 36: 1348-1354.

38. Han CS, Mulla MJ, Brosens JJ, Chamley LW, Paidas MJ, Lockwood CJ, et al. Aspirin and heparin effect on basal and antiphospholipid antibody modulation of trophoblast function. Obstet Gynecol 2011; 118: 1021-1028, doi: 10.1097/ AOG.0b013e31823234ad.

39. Fawzy M, Shokeir T, El-Tatongy M, Warda O, El-Refaiey AAA, Mosbah A. Treatment options and pregnancy outcome in women with idiopathic recurrent miscarriage: a randomized placebo-controlled study. Arch Gynecol Obstet 2008; 278: 33-38, doi: 10.1007/s00404-007-0527-x. 